\section{Percepção estética de \\ fluorose dentária entre jovens \\ universitários}

\section{Aesthetic perceptions of dental fluorosis among young university students}

Márcia Helena Baldani ${ }^{1}$

Paula Fabiane Fonseca Araújo'

Denise Stadler Wambier ${ }^{1}$

Maryana Luzia Strosky ${ }^{1}$

Célia Maria Da Lozzo Lopes ${ }^{2}$

${ }^{1}$ Departamento de Odontologia da Universidade Estadual de Ponta Grossa UEPG

${ }^{2}$ Departamento de Biologia e Genética da Universidade Estadual de Ponta Grossa - UEPG

\section{Resumo}

Objetivo: Identificar os níveis de aprovação da fluorose dentária entre adultos leigos e cirurgiões-dentistas, comparando os padrões de aceitação da aparência de dentes afetados por acadêmicos da área de Ciências Exatas, representando a população leiga, com os de formandos do curso de Odontologia, representando os profissionais. Método: Participaram 180 alunos dos cursos de Odontologia e da área das Ciências Exatas, os quais responderam a questionário acerca de fotografias de arcadas dentárias com fluorose nos níveis muito leve, leve e moderada de Dean, e moderada após aplicação da técnica de micro-abrasão do esmalte. Perguntou-se sobre aparência geral da boca; coloração, forma e posição dos dentes; aspecto da gengiva e possibilidade de sorrir sem constrangimento. A análise dos dados foi realizada utilizando-se o teste qui-quadrado e regressão logística com intervalos de confiança em nível de 95\%. Resultados: Houve maior percentual de aprovação quanto à aparência da boca $\mathrm{e}$ coloração dos dentes para o caso de fluorose muito leve. Para todos os casos os alunos dos cursos de Exatas mostraram-se mais exigentes do que os acadêmicos de Odontologia. Verificou-se associação significativa entre percepção de aparência da boca e coloração dos dentes. A análise multivariada revelou que, para o caso de fluorose muito leve, a coloração dos dentes foi incluída no modelo explicativo de percepção da aparência da boca apenas para o grupo dos formandos. Conclusões: Todos os níveis de fluorose foram percebidos pelos grupos estudados, porém os casos de fluorose muito leve parecem não comprometer a percepção positiva da aparência da boca para os grupos que representam indivíduos leigos.

Palavras-chave: Estética dentária. Fluorose dentária. Percepção. 


\section{Abstract}

Objective: To identify the levels of approval of dental fluorosis among lay adults and dentists, comparing the standards of acceptance of the appearance of affected teeth among groups of university students attending the area of Exact Sciences, representing the lay population, and senior Dentistry students, representing dentists. Methods: 180 students participated by answering questions concerning dental arch photographs, with Dean's very mild, mild and moderate fluorosis index, and moderate fluorosis after the application of the microabrasion enamel technique. The questionnaire comprised questions about the general appearance of the mouth; coloration, form and position of teeth; aspect of the gums and the possibility of smiling without embarrassment. Data was analyzed using the Chi-square test and reliable logistic regression with $95 \%$ confidence intervals. Results: There were higher approval ratios, related to the appearance of the mouth and coloration of teeth, for the cases of very mild fluorosis. For all cases, Exact Sciences students were more demanding than Dentistry students. There was a significant association between perceived appearance of the mouth and discoloration of the teeth. The multivariate analysis revealed that, in the case of very mild fluorosis, the discoloration of teeth was included in the explanatory model of the perception of the appearance of the mouth only for the group of senior Dentistry students. Conclusions: All levels of fluorosis were perceived by the groups studied; however the cases of very mild fluorosis do not seem to compromise the positive perception of the appearance of the mouth, for the groups that represent lay individuals.

Keywords: Dental aesthetic. Dental fluorosis. Perception.

\section{Introdução}

Dentre os métodos preventivos utilizados em saúde pública destacam-se os que se ajustam às denominadas estratégias populacionais, devido ao impacto que têm sobre os níveis de agravos nas populações ${ }^{1,2}$. A cárie dentária está em declínio no mundo, e também no Brasil, e este declínio é atribuído principalmente à larga utilização de fluoretos pela água de abastecimento público e por dentifrícios, ou seja, por intermédio de medidas de abrangência populacional ${ }^{3,4}$. Dentre as principais medidas de oferta de flúor para a redução dos níveis de cárie na população destaca-se a fluoretação das águas de abastecimento público, por se verificar que o maior impacto desta ocorre onde as condições sociais são piores e a população não tem acesso a outros meios de proteção ${ }^{5-7}$.

Porém, apesar dos comprovados benefícios dos fluoretos para a prevenção da cárie, não se pode descartar a presença de riscos decorrentes de sua utilização, uma vez que a ingestão constante de doses acima das consideradas seguras, no período de desenvolvimento dos dentes, pode levar ao desenvolvimento de fluorose dentária. Tem sido observada uma tendência ao aumento da prevalência de fluorose ${ }^{8}$, o qual tem se manifestado principalmente nas formas menos graves, sendo verificado tanto em comunidades com água de abastecimento fluoretada quanto naquelas que não a possuem $^{8}$.

Segundo o mais recente levantamento epidemiológico em saúde bucal realizado pelo Ministério da Saúde, a prevalência de fluorose dentária no Brasil é de cerca de $9 \%$ em crianças de 12 anos e de $5 \%$ em adolescentes de 15 a 19 anos, considerando a população residente em municípios com e sem água de abastecimento público fluoretada. Para a idade de 12 anos, os maiores índices foram encontrados nas Regiões Sudeste e Sul (em torno de 12\%), enquanto os menores ocorreram nas Regiões CentroOeste e Nordeste (cerca de $4 \%)^{9}$.

Historicamente, os benefícios advindos 
da redução da cárie e o risco para a fluorose são interligados, sendo que, em saúde pública, uma prevalência baixa de formas leves de sua manifestação tem sido considerada aceitável em relação à significativa proteção conferida pela medida ${ }^{10}$. Com a ampliação dos métodos para a utilização de fluoretos, principalmente com a inserção no mercado dos dentifrícios fluoretados, clínicos e sanitaristas têm voltado sua atenção para os riscos de um aumento inaceitável de prevalência da fluorose na população, inclusive nas formas mais graves, o que tem levado a discussões sobre a necessidade de controle dos teores de flúor na água, alimentos, bebidas e produtos de higiene, além de orientação da população quanto aos cuidados para sua prevenção ${ }^{11}$.

Moysés et al. ${ }^{11}$ comentam que, diante da possibilidade de aumento da prevalência da fluorose no Brasil, estudos adicionais são necessários para determinar quando e em que nível a fluorose dentária tem um efeito negativo, autopercebido pela população.

A percepção em saúde bucal está associada aos aspectos físicos e subjetivos relacionados à boca, e é influenciada por fatores sociais e econômicos, por idade, sexo e classe social do indivíduo ${ }^{12}$. Estudos dirigidos para a identificação da percepção estética da população quanto à fluorose dentária, envolvendo principalmente pais e crianças na faixa etária de 12-15 anos, constataram que as formas menos graves são pouco percebidas e esteticamente aceitáveis ${ }^{10,11,13-18}$.

Tem sido verificado que indivíduos leigos, portadores ou não, tendem a identificar as formas menos graves de fluorose como menos aceitáveis do que os dentistas ${ }^{10,19,20}$. Estudos como os de Clark ${ }^{21}$ (1995), Clark e Berkowitz ${ }^{22}$ (1997), e de Shulman et al. ${ }^{20}$ (2004) identificaram que as crianças são mais críticas quanto à coloração dos dentes do que seus pais, os quais, por sua vez, mostram-se mais críticos do que os dentistas. Martins et al. ${ }^{18}$ concluíram que manchas de fluorose, mesmo em graus mais leves, são percebidas por pessoas leigas e, na maioria das vezes, elas as julgam prejudiciais.
Shulman et al. ${ }^{20}$ estudaram a aprovação da coloração dos dentes por crianças e adolescentes, e compararam com a de seus pais e dentistas. Dentre os indivíduos que se mostraram insatisfeitos com a coloração dos dentes (31,6\%), 11,7\% citaram a fluorose como razão da insatisfação. Esta foi citada por $12,5 \%$ dos pais e por apenas $8,5 \%$ dos dentistas. Levy et al. ${ }^{23}$ compararam a percepção de fluorose dentária e opacidades de esmalte entre estudantes de Odontologia no primeiro (ainda leigos) e no último ano da graduação. Observaram que, no final do curso, os acadêmicos apresentaram maior disposição em atribuir notas favoráveis às imagens apresentadas do que no início do curso.

Buscando contribuir com informações que auxiliem a conhecer os aspectos psicossociais relacionados com a percepção estética da fluorose dentária, o objetivo deste estudo foi identificar os níveis de aprovação entre adultos leigos e cirurgiões-dentistas, comparando os padrões de aceitação da aparência dos dentes afetados por acadêmicos da área de Ciências Exatas, representando a população leiga, com os de formandos do curso de Odontologia, representando os profissionais.

\section{Métodos}

Delineou-se um estudo de corte transversal, exploratório, do qual participaram 180 acadêmicos da Universidade Estadual de Ponta Grossa (UEPG), alocados em dois grupos: 90 acadêmicos do primeiro ano de cursos da área de Ciências Exatas (Química e Engenharia de Materiais); e 90 acadêmicos formandos do curso de Odontologia. O primeiro grupo foi constituído com o objetivo de identificar a percepção de adultos jovens leigos quanto à fluorose dentária. A partir do grupo de formandos buscou-se obter uma aproximação da percepção dos cirurgiõesdentistas. Não houve, em nenhum momento da pesquisa, conflito de interesses por parte dos autores.

A amostra, de conveniência, envolveu todos os acadêmicos matriculados no 
último ano do curso de Odontologia, em 2006 e 2007, e no primeiro ano dos cursos de Química e Engenharia de Materiais que concordaram em participar da pesquisa. Após assinarem o termo de consentimento livre e esclarecido, conforme aprovado pelo Comitê de Ética em Pesquisa da instituição, os acadêmicos foram convidados a observar uma seqüência de quatro fotografias intrabucais frontais de dentes com fluorose nos graus muito leve, leve e moderada, $\mathrm{e}$ moderada, após aplicação da técnica de micro-abrasão do esmalte dentário. Após cada observação, solicitou-se que respondessem às perguntas descritas no Quadro 1 . As fotografias selecionadas para a pesquisa foram previamente analisadas por três profissionais experientes em levantamentos epidemiológicos de fluorose dentária, aos quais se recomendou que classificassem o nível de comprometimento dos dentes anteriores empregando o índice de Dean ${ }^{24}$. As arcadas dentárias fotografadas apresentavam dentes em posição de oclusão normal, ou com alterações discretas. $\mathrm{O}$ aspecto da gengiva era normal para a maioria das imagens, com pequenos sinais de inflamação em sítios isolados.

As fotografias foram apresentadas aos sujeitos da pesquisa por meio de slides, em sala escurecida, com imagem ocupando completamente uma tela de projeção de cerca de 1 metro de altura, formando-se grupos pequenos para permitir distâncias semelhantes de todos à tela de projeção. A seqüência de imagens, mantida constante para todos os grupos, foi organizada segundo níveis crescentes de gravidade de fluorose dentária. Para cada imagem projetada perguntou-se sobre a aparência geral da boca; coloração, forma e posição dos dentes; aspecto da gengiva e possibilidade de sorrir sem constrangimento (Quadro 1), utilizando-se metodologia adaptada de McKnight et al. ${ }^{15}$ e de Levy et al. ${ }^{23}$. Procurando-se evitar viés de aferição, não foram feitos comentários prévios sobre fluorose, cor ou manchas nos dentes apresentados. A metodologia da pesquisa foi submetida a pré-teste por meio de estudo piloto envolvendo 52 acadêmicos do segundo ano de Odontologia da UEPG.

As informações obtidas foram analisadas pelo programa SPSS for Windows versão 15.0 (SPSS Inc. Headquarters, Chicago, Illinois). Na análise dos dados empregou-se o teste qui-quadrado, bem como o cálculo de odds ratio, com intervalos de confiança em nível de 95\%, estimados por meio de regressão logística. Para fins de análise de associação, a variável de percepção da aparência da boca foi transformada em dicotômica, considerando-se: aparência/ coloração aceitável - soma dos escores 3 (aceitável) e 4 (excelente); e aparência/ coloração inaceitável - os demais escores

Quadro 1 - Questionário referente às fotografias de arcos dentários com fluorose.

Chart 1 - Questionnaire of photographs of dental arches with fluorosis.

1 - Qual a sua opinião quanto à aparência desta boca?

( ) péssima (0) ( ) ruim (1) () regular (2) ( ) aceitável (3) ( ) excelente (4)

2 - Qual a sua opinião quanto à coloração dos dentes?

() péssima (0) () ruim (1) () regular (2) () aceitável (3) () excelente (4)

3 - Qual a sua opinião quanto à forma e posição dos dentes?

( ) péssima (0) ( ) ruim (1) ( ) regular (2) ( ) aceitável (3) ( ) excelente (4)

4 - Qual a sua opinião quanto ao aspecto da gengiva?

( ) péssima (0) () ruim (1) () regular (2) ( ) aceitável (3) ( ) excelente (4)

5 - Suponha que estes dentes são seus, você sorriria deixando-os à mostra?

( ) nunca (0) ( ) quase nunca (1) ( ) às vezes (2) ( ) sempre (3) 
(Quadro 1). Também para a variável de freqüência com que sorriria, as respostas foram organizadas em duas categorias: às vezes/sempre; e nunca/quase nunca. Para aferir se a satisfação demonstrada quanto à aparência da boca estava relacionada à presença de fluorose (coloração dos dentes), os modelos multivariados foram controlados pela percepção de aspecto da gengiva e forma/posição dos dentes.

\section{Resultados}

Participaram do estudo 180 acadêmicos. Os dois grupos constituídos, apresentaramse homogêneos quanto ao número de indivíduos (90 formandos de Odontologia e 90 acadêmicos da área das Ciências Exatas). Com relação ao gênero, o grupo de formandos de Odontologia apresentou uma proporção maior de mulheres (54\%) do que o de acadêmicos da área das Ciências Exatas (40\%), sendo a diferença estatisticamente significante $(\mathrm{p}=0,05)$.

Verifica-se, na Tabela 1 que a proporção de aprovação da coloração dos dentes, aparência da boca e freqüência com que sorriria deixando os dentes visíveis diminui conforme aumenta a gravidade da fluorose dentária. Esta característica é mais acentuada para a variável coloração dos dentes, que está mais diretamente relacionada com os aspectos clínicos deste agravo. Observa-se também que os acadêmicos da área das Ciências Exatas foram os que menos demonstraram aprovar as imagens, e as diferenças de percepção entre os grupos foram significativas para os casos menos graves.

Na Tabela 2, observa-se que não houve diferença significativa entre os gêneros, sen-

Tabela 1 - Distribuição proporcional dos universitários para a percepção de coloração dos dentes e aparência da boca, além de freqüência com que sorriria, segundo as turmas $(n=180)$.

Table 1 - Proportional distribution of university students as to the perception of discoloration of teeth and appearance of the mouth, and how often they would smile, according to groups $(n=180)$

\begin{tabular}{|c|c|c|c|c|c|c|c|c|c|}
\hline \multirow[t]{2}{*}{ Turma } & \multicolumn{3}{|c|}{$\begin{array}{l}\text { Coloração dos dentes } \\
\text { aceitável/ excelente }\end{array}$} & \multicolumn{3}{|c|}{$\begin{array}{c}\text { Aparência da boca aceitável/ } \\
\text { excelente }\end{array}$} & \multicolumn{3}{|c|}{ Sorriria às vezes/ sempre } \\
\hline & $\mathrm{n}$ & $\%$ & $\mathrm{p}$-valor* & $\mathrm{n}$ & $\%$ & $\mathrm{p}$-valor* & $\mathrm{n}$ & $\%$ & $\mathrm{p}$-valor* \\
\hline Foto 1 (muito leve) & & & $<0,001$ & & & $<0,001$ & & & 0,109 \\
\hline Formandos & 64 & 71,1 & & 63 & 70,0 & & 79 & 87,8 & \\
\hline Exatas & 35 & 38,9 & & 34 & 37,8 & & 71 & 78,9 & \\
\hline Total & 94 & 52,2 & & 97 & 53,9 & & 150 & 83,3 & \\
\hline Foto 2 (leve) & & & $0,014^{* *}$ & & & 0,002 & & & $<0,001$ \\
\hline Formandos & 6 & 6,7 & & 11 & 12,2 & & 58 & 64,4 & \\
\hline Exatas & 0 & 0 & & 0 & 0 & & 28 & 31,1 & \\
\hline Total & 6 & 3,3 & & 11 & 6,1 & & 86 & 47,8 & \\
\hline Foto 3 (moderada) & & & $0,500^{* *}$ & & & $0,221^{* *}$ & & & 0,473 \\
\hline Formandos & 0 & 0 & & 5 & 5,6 & & 22 & 24,4 & \\
\hline Exatas & 1 & 1,1 & & 2 & 2,2 & & 18 & 20,0 & \\
\hline Total & 1 & 0,6 & & 7 & 3,9 & & 40 & 22,2 & \\
\hline $\begin{array}{l}\text { Foto } 4 \text { (moderada após micro- } \\
\text { abrasão) }\end{array}$ & & & 0,197 & & & 0,014 & & & 0,004 \\
\hline Formandos & 22 & 24,4 & & 43 & 47,8 & & 75 & 83,3 & \\
\hline Exatas & 15 & 16,7 & & 27 & 30,0 & & 58 & 64,4 & \\
\hline Total & 37 & 20,5 & & 70 & 38,9 & & 133 & 73,9 & \\
\hline
\end{tabular}

* Teste qui-quadrado ** Teste exato de Fisher

* Chi-square test ${ }^{* *}$ Fisher's exact test 
do que, tanto para os homens quanto para as mulheres, a aprovação da aparência da boca e da coloração dos dentes, bem como a freqüência com que sorririam diminui conforme aumenta o nível de gravidade da fluorose dentária.

Para os níveis menos graves de fluorose, bem como para a imagem de fluorose moderada após micro-abrasão do esmalte, houve associação significativa entre a percepção positiva da aparência da boca com a melhor aceitação da coloração dos dentes para ambos os grupos (Tabela 3), havendo diferenças quanto ao nível de aceitação da imagem de fluorose leve.

As imagens que apresentaram associação significativa entre a aparência da boca e a coloração dos dentes foram selecionadas para a análise multivariada por turma (Tabela 4), com o objetivo de identificar o melhor modelo explicativo para a percepção positiva de aparência da boca em cada grupo, incluindo-se, além da coloração dos dentes, a forma e posição dos mesmos e a aparência da gengiva. Observa-se que, para o caso de fluorose muito leve, a coloração dos dentes foi incluída no modelo explicativo apenas para o grupo dos formandos, sendo que, para os acadêmicos da área das Ciências Exatas, a característica que explicou a satisfação com a aparência da boca foram forma e posição dos dentes. Para os casos mais graves de fluorose, a coloração dos dentes foi a única variável significativa para ambos os grupos de universitários.

\section{Discussão}

Diversos estudos têm demonstrado que os atrativos físicos são psicologicamente

Tabela 2 - Distribuição proporcional dos universitários para a percepção de coloração dos dentes e aparência da boca, além de freqüência com que sorriria, segundo o gênero $(n=172)$.

Table 2 - Proportional distribution of university students as to the perception of discoloration of teeth and appearance of the mouth, and how often they would smile, according to gender $(n=172)$

\begin{tabular}{|c|c|c|c|c|c|c|c|c|c|}
\hline \multirow[t]{2}{*}{ Gênero } & \multicolumn{3}{|c|}{$\begin{array}{c}\text { Coloração dos dentes aceitável/ } \\
\text { excelente }\end{array}$} & \multicolumn{3}{|c|}{$\begin{array}{c}\text { Aparência da boca aceitável/ } \\
\text { excelente }\end{array}$} & \multicolumn{3}{|c|}{ Sorriria às vezes/ sempre } \\
\hline & $\mathrm{n}$ & $\%$ & p-valor* & $\mathrm{n}$ & $\%$ & $\mathrm{p}$-valor* & $\mathrm{n}$ & $\%$ & $\mathrm{p}$-valor* \\
\hline Foto 1 (muito leve) & & & 0,936 & & & 0,244 & & & 0,442 \\
\hline Masculino & 50 & 54,9 & & 47 & 51,6 & & 78 & 83,9 & \\
\hline Feminino & 45 & 55,6 & & 49 & 60,5 & & 71 & 87,7 & \\
\hline Total & 95 & 55,2 & & 96 & 55,8 & & 152 & 83,5 & \\
\hline Foto 2 (leve) & & & $0,287^{* *}$ & & & 0,173 & & & 0,373 \\
\hline Masculino & 2 & 2,2 & & 8 & 8,8 & & 42 & 45,2 & \\
\hline Feminino & 4 & 4,9 & & 3 & 3,7 & & 42 & 51,9 & \\
\hline Total & 6 & 3,5 & & 11 & 6,4 & & 88 & 48,3 & \\
\hline Foto 3 (moderada) & & & $0,268^{* * *}$ & & & $0,272^{* *}$ & & & 0,544 \\
\hline Masculino & 1 & 1,1 & & 5 & 5,5 & & 20 & 21,5 & \\
\hline Feminino & 3 & 3,7 & & 2 & 2,5 & & 21 & 25,9 & \\
\hline Total & 1 & 0,6 & & 7 & 4,1 & & 42 & 23,1 & \\
\hline $\begin{array}{l}\text { Foto } 4 \text { (moderada após } \\
\text { micro-abrasão) }\end{array}$ & & & 0,063 & & & 0,878 & & & 0,506 \\
\hline Masculino & 24 & 26,4 & & 33 & 36,6 & & 69 & 74,2 & \\
\hline Feminino & 12 & 14,8 & & 34 & 42,0 & & 64 & 79,0 & \\
\hline Total & 36 & 20,9 & & 67 & 39,0 & & 135 & 74,2 & \\
\hline
\end{tabular}

* Teste qui-quadrado **Teste exato de Fisher

* Chi-square test ${ }^{* *}$ Fisher's exact test 
Tabela 3 - Análise da associação entre a percepção da aparência da boca e da coloração dos dentes apresentados nas fotografias, para os grupos de universitários $(n=180)$.

Table 3 - Analysis of the association between perception of the appearance of the mouth and discoloration of teeth presented in photographs, for the groups of university students $(n=180)$

\begin{tabular}{|c|c|c|c|c|c|c|c|c|c|c|}
\hline \multirow{3}{*}{ Nível de fluorose } & \multicolumn{5}{|c|}{ Formandos $(n=90)$ Aparência da boca } & \multicolumn{5}{|c|}{ Exatas $(n=90)$ Aparência da boca } \\
\hline & \multicolumn{2}{|c|}{ aceitável } & \multicolumn{2}{|c|}{ inaceitável } & \multirow{2}{*}{ p-valor* } & \multicolumn{2}{|c|}{ aceitável } & \multicolumn{2}{|c|}{ inaceitável } & \multirow{2}{*}{ p-valor* } \\
\hline & $\mathrm{n}$ & $\%$ & $\mathrm{n}$ & $\%$ & & $\mathrm{n}$ & $\%$ & $\mathrm{n}$ & $\%$ & \\
\hline \multicolumn{11}{|l|}{ Foto 1 (muito leve) } \\
\hline Cor dos dentes & & & & & $<0,001$ & & & & & $<0,001$ \\
\hline Aceitável & 52 & 81,3 & 12 & 18,8 & & 21 & 60,0 & 14 & 40,0 & \\
\hline Regular & 10 & 45,5 & 12 & 54,5 & & 12 & 30,8 & 27 & 69,2 & \\
\hline Inaceitável & 1 & 25,0 & 3 & 75,0 & & 1 & 6,3 & 15 & 93,8 & \\
\hline \multicolumn{11}{|l|}{ Foto 2 (leve) } \\
\hline Cor dos dentes & & & & & $<0,001$ & & & & & - \\
\hline Aceitável & 4 & 66,7 & 2 & 33,3 & & & & & & \\
\hline Regular & 4 & 15,4 & 22 & 84,6 & & 0 & 0,0 & 5 & 100,0 & \\
\hline Inaceitável & 3 & 5,2 & 55 & 94,8 & & 0 & 0,0 & 85 & 100,0 & \\
\hline \multicolumn{11}{|l|}{ Foto 3 (moderada) } \\
\hline Cor dos dentes & & & & & $0,425^{* *}$ & & & & & 0,407 \\
\hline Aceitável & & & & & & 0 & 0,0 & 1 & 100,0 & \\
\hline Regular & 0 & 0,0 & 7 & 100,0 & & 1 & 25,0 & 3 & 75,0 & \\
\hline Inaceitável & 10 & 12,0 & 73 & 88,0 & & 6 & 7,1 & 79 & 92,9 & \\
\hline \multicolumn{11}{|c|}{ Foto 4 (moderada após micro-abrasão) } \\
\hline Cor dos dentes & & & & & $<0,001$ & & & & & $<0,001$ \\
\hline Aceitável & 20 & 90,9 & 2 & 9,1 & & 13 & 86,7 & 2 & 13,3 & \\
\hline Regular & 18 & 52,9 & 16 & 47,1 & & 6 & 35,3 & 11 & 64,7 & \\
\hline Inaceitável & 5 & 14,7 & 29 & 85,3 & & 8 & 13,8 & 50 & 86,2 & \\
\hline
\end{tabular}

* Teste qui-quadrado **Teste exato de Fisher

* Chi-square test ${ }^{* *}$ Fisher's exact test

importantes, em especial para crianças e adultos jovens ${ }^{13}$. Papel primordial na determinação destes é atribuído à face, sendo a região bucal a principal determinante de um rosto atraente ${ }^{13}$. Dessa forma, mostramse necessárias pesquisas que identifiquem a autopercepção dos indivíduos quanto à aparência de seus dentes, bem como a percepção de outras pessoas. No caso da fluorose dentária, torna-se imprescindível verificar o quanto a sua presença, nos níveis menos severos, é percebida pela população e impacta a qualidade de vida dos indivíduos portadores, não apenas sob o ponto de vista clínico mas, principalmente, sob o ponto de vista estético.
Como demonstrado por outros estu$\operatorname{dos}^{10,16,17,19,21,25}$, os indivíduos leigos foram capazes de distinguir entre os diferentes níveis de fluorose. Para ambos os grupos de universitários, houve maior percentual de aprovação quanto à aparência da boca e coloração dos dentes para as imagens de fluorose muito leve e moderada após micro-abrasão do esmalte. No entanto, os acadêmicos dos cursos de Exatas mostraram-se mais exigentes em todos os aspectos analisados.

Não foram observadas diferenças significativas quanto ao gênero. Resultados semelhantes foram obtidos por Hawley et al. ${ }^{16}$, que não identificaram diferenças en- 
Tabela 4 - Análise de regressão logística multivariada para fluorose no nível muito leve e leve de Dean e moderada após micro-abrasão do esmalte dentário. Modelos explicativos para a percepção da aparência da boca para os dois grupos de universitários*.

Table 4 - Multivariate logistic regression analysis for Dean's very mild and mild fluorosis index, and moderate level of fluorosis after micro abrasion of the dental enamel. Explanatory models of the perception of the appearance of the mouth for the two groups of university students*

\begin{tabular}{|c|c|c|c|c|c|c|}
\hline \multirow[t]{2}{*}{ Imagem } & \multicolumn{3}{|c|}{ Formandos $(n=90)$} & \multicolumn{3}{|c|}{ Exatas $(n=90)$} \\
\hline & Modelo & $\mathrm{OR}_{\mathrm{aj}}(\mathrm{IC} 95 \%)$ & $\mathrm{p}$ & Modelo & $\mathrm{OR}_{\mathrm{aj}}(\mathrm{IC} 95 \%)$ & $\mathrm{p}$ \\
\hline Foto 1 Muito & & $\mathrm{R} 2=0,29$ & & & $\mathrm{R} 2=0,37$ & \\
\hline \multirow[t]{12}{*}{ Leve } & Cor dos dentes & & 0,004 & Cor dos dentes & & NS \\
\hline & Aceitável & 1,00 & & & & \\
\hline & Regular & $0,06(0,00-1,00)$ & & & & \\
\hline & Inaceitável & $0,43(0,03-7,14)$ & & & & \\
\hline & $\begin{array}{l}\text { Forma e posição dos } \\
\text { dentes }\end{array}$ & & 0,014 & $\begin{array}{c}\text { Forma e posição dos } \\
\text { dentes }\end{array}$ & & 0,021 \\
\hline & Aceitável & 1,00 & & Aceitável & 1,00 & \\
\hline & Regular & $0,16(0,04-0,71)$ & & Regular & $0,05(0,00-0,48)$ & \\
\hline & Inaceitável & $0,16(0,04-0,63)$ & & Inaceitável & $0,05(0,00-0,42)$ & \\
\hline & Aspecto da gengiva & & 0,039 & Aspecto da gengiva & & 0,095 \\
\hline & Aceitável & 1,00 & & Aceitável & 1,00 & \\
\hline & Regular & $0,15(0,03-0,64)$ & & Regular & $0,09(0,01-0,84)$ & \\
\hline & Inaceitável & $0,37(0,09-1,55)$ & & Inaceitável & $0,08(0,01-0,88)$ & \\
\hline
\end{tabular}

Foto 2 Leve

$\mathrm{R} 2=0,22$

Cor dos dentes

0,039

Aceitável $\quad 1,00$

Regular $\quad 0,03(0,00-0,46)$

Inaceitável $\quad 0,40(0,08-2,14)$

Forma e posição dos dentes

NS

Aspecto da gengiva

NS

Foto 4 Moderada após micro abrasão

$\begin{array}{cc}\text { Cor dos dentes } & \mathrm{R} 2=0,37 \\ \text { Aceitável } & 1,00 \\ \text { Regular } & 0,02(0,00-0,12) \\ \text { Inaceitável } & 0,18(0,05-0,60)\end{array}$

Forma e posição dos dentes

NS $<0,001$

Cor dos dentes $\mathrm{R} 2=0,33$

Aceitável 1,00

Aspecto da gengiva

NS Aspecto da gengiva

NS

NS

* Variáveis testadas: coloração dos dentes; forma e posição dos dentes; aspecto da gengiva.

* Tested variables: discoloration of the teeth; shape and position of teeth; aspect of gums.

NS - não significativo ao nível de 5\%. NS - non significant to the 5\% level

tre meninos e meninas de 14 anos quanto à aprovação estética de dentes anteriores com fluorose dentária. Por outro lado, Shulman et al. ${ }^{20}$ encontraram, em uma amostra de 2.495 crianças e adolescentes, que as meninas foram mais exigentes quanto à coloração de seus dentes do que os meninos. Os pais e dentistas, por sua 
vez, foram mais exigentes quanto à coloração dos dentes dos meninos.

Neste estudo, a maior aprovação dos acadêmicos para os casos de fluorose muito leve assemelha-se a pesquisas que têm buscado identificar a percepção estética da fluorose dentária envolvendo pais, crianças e dentistas ${ }^{10,11,14-18,19}$. Nestes, tem sido observado que as formas menos graves são pouco percebidas e esteticamente aceitáveis, e que o nível de aprovação diminui à medida que aumenta a gravidade da fluorose.

$\mathrm{O}$ fato de os acadêmicos da área das Ciências Exatas mostrarem-se mais exigentes está de acordo com as pesquisas direcionadas à comparação entre a percepção de profissionais e leigos. Nestas foi demonstrado que indivíduos leigos, portadores ou não, tendem a identificar as formas menos graves de fluorose como menos aceitáveis do que os dentistas, e também que todos são capazes de distinguir entre dentes com e sem fluorose ${ }^{10,19,20,22,23,25,}$. No entanto, verificou-se também que, apesar da percepção negativa, boa parte dos indivíduos, de ambas as turmas, sorriria normalmente se apresentasse o problema (Tabela 1).

Shulman et al. ${ }^{20}$ apresentam algumas explicações possíveis para o fato da fluorose dentária menos severa ser considerada como mais aceitável pelos dentistas do que pelos indivíduos leigos: os dentistas associam fluorose com exposição ao flúor, o qual eleva a resistência dos dentes contra a cárie dentária, e isso faz com que esta seqüela seja vista como conseqüência natural de uma medida preventiva desejável; os dentistas são expostos à grande diversidade de problemas bucais mais graves em sua clínica diária; estes têm conhecimentos sobre as causas do problema e as limitações quanto às possibilidades de tratamento, enquanto os pacientes identificam apenas o problema estético.

Para os casos menos graves de fluorose (muito leve e leve), bem como para o de fluorose moderada após aplicação da técnica da micro-abrasão do esmalte dentário, verificou-se associação significativa entre percepção positiva de aparência da boca com a coloração dos dentes. Para o caso de fluorose muito leve, a análise multivariada selecionou a coloração dos dentes como variável explicativa para percepção positiva da aparência da boca, apenas para o grupo dos formandos, não sendo significativa para o grupo dos acadêmicos das áreas das Ciências Exatas, que representam indivíduos leigos neste estudo e foram mais críticos quanto à aprovação das imagens. Estes resultados parecem confirmar estudos realizados com pais e indivíduos portadores. O estudo de Lalumandier e Rozier ${ }^{13}$ indicou que os pais só se mostraram insatisfeitos com a coloração dos dentes no caso de crianças portadoras de fluorose dentária nos níveis mais severos. Estudos como os de Moysés et al. ${ }^{11}$, e de Peres et al. ${ }^{14}$, entre outros, tem demonstrado que a satisfação com a aparência da boca, entre indivíduos de 12 anos portadores, está associada com outras variáveis e não com a fluorose dentária. O estudo de Shulman et al..$^{20}$ identificou que, tanto para crianças, quanto para seus pais e dentistas, a principal razão para a insatisfação com a aparência dos dentes foi a coloração amarelada destes, sendo a fluorose pouco citada.

Ainda quanto ao caso de fluorose muito leve, diferentemente da coloração dos dentes, a forma e a posição destes foram incluídas no modelo multivariado tanto para os acadêmicos de Odontologia quanto para os de Exatas. Segundo Shulman et al. ${ }^{20}$, a percepção da oclusão de dentes anteriores difere daquela da coloração dos dentes. Para estes casos, os dentistas mostram-se mais críticos do que indivíduos leigos. No entanto, Pietilä e Pietilä ${ }^{26}$ encontraram concordância de $60 \%$ entre a percepção de maloclusão por ortodontistas e pais, mais freqüente quando esta afetava os dentes anteriores.

Comparando-se as respostas para as imagens de dentes com fluorose moderada (com e sem tratamento), obteve-se maior proporção de aprovação após a microabrasão do esmalte dentário, apesar do procedimento não se mostrar capaz de eliminar completamente os traços fluoróticos dos 
dentes. Segundo Lynch e McConnel ${ }^{27}$, esta técnica promove uma camada superficial de esmalte que reflete e refrata a luz de maneira diferente do esmalte não tratado, camuflando as manchas subjacentes, o que leva a uma aparência mais aceitável.

Alguns fatores devem ser considerados quando da interpretação dos resultados aqui descritos. O primeiro deles refere-se às características da amostra, de conveniência, envolvendo adultos jovens com alto nível de escolaridade, os quais correspondem a uma pequena parcela da população adulta. Quanto a este fato, Lalumandier e Rozier ${ }^{13}$ verificaram, em seu estudo, que o nível de escolaridade dos pais não influenciou sua percepção quanto à coloração dos dentes de seus filhos. Também não foram obtidas informações sobre características socioeconômicas para a população estudada. Segundo os relatórios dos concursos vestibulares de 2006 e 2007 da UEPG, a faixa de renda predominante para os cursos incluídos no estudo esteve entre 3 e 15 salários mínimos. Porém, Shulman et al. ${ }^{20}$ não encontraram associação significativa entre melhor ou pior condição socioeconômica dos pais e o nível de exigência quanto à coloração dos dentes dos filhos.

Outro aspecto que poderia afetar as respostas seria, segundo McKnight et al. ${ }^{25}$, o fato de as fotografias terem sido obtidas de diferentes pacientes, com diferentes tamanhos, formas e posições dos dentes nas arcadas, além de distintas condições gengivais. No entanto, em estudo recente no qual um grupo de adolescentes foi apresentado a imagens de uma boca com coloração dos dentes modificada por simulação digital para apresentar fluorose nos vários níveis de gravidade, apresentaram resultados semelhantes aos aqui observados: os indivíduos puderam identificar os vários níveis de fluorose, e a aceitação diminuiu quanto maior foi o grau de severidade ${ }^{17}$. Nesse mesmo estudo, os autores identificaram que a per- cepção estética positiva (aceitação) para as fotografias intrabucais é menor do que para as imagens que apresentam os dentes no contexto do rosto (em um sorriso, por exemplo), e também está associada à distância da observação. Concluíram que as imagens em "closes" intrabucais podem afetar adversamente a percepção estética. Uma vez que as fotografias utilizadas na presente pesquisa apresentavam imagens intrabucais e com dimensões ampliadas, deve-se considerar que os resultados obtidos podem estar superestimados. Também não se pode descartar a possibilidade de que o impacto maior das fotografias sobre os alunos dos cursos de Exatas tenha ocorrido pela pouca familiaridade destes com as imagens intrabucais.

Finalmente, não foram consideradas as "experiências" dos sujeitos da pesquisa relacionadas às condições apresentadas. Supõe-se que os indivíduos portadores de fluorose ou de maloclusões, por exemplo, possam apresentar um padrão de respostas diferente daqueles que não possuem estas patologias. Esta dimensão poderia ser melhor compreendida por meio de estudos qualitativos, que dêem conta de identificar as representações sociais dos indivíduos quanto à fluorose dentária.

\section{Conclusões}

Todos os níveis de fluorose foram percebidos pelos grupos estudados, porém os casos de fluorose muito leve parecem não comprometer a percepção positiva de aparência da boca para os grupos que representam indivíduos leigos. As características aqui relatadas sugerem a necessidade de estudos adicionais que considerem o impacto biopsicossocial da fluorose dentária em população de adultos jovens com alta escolaridade, uma vez que, neste período da vida, os indivíduos estão em intensa atividade social e profissional, e as necessidades estéticas encontram-se acentuadas. 


\section{Referências}

1. Burt BA. Concepts of risk in dental public health. Community Dent Oral Epidemiol 2005; 33: 240-7.

2. Batchelor P, Sheiham A. The distribution of burden of dental caries in schoolchildren: a critique of the highrisk caries prevention strategy for populations. BMC Oral Health 2006; 6. Periódico on-line. Disponível em: http:/www.biomedcentral.com.

3. Peterson HG, Bratthall D. The caries decline: a review of reviews. Eur J Oral Science 1996; 104: 436-43.

4. McDonagh MS, Whiting PF, Wilson PM, Sutton AJ, Chestnutt I, Coope J et al. Systematic review of water fluoridation. Br Med J 2000, 321: 855-9.

5. Slade GD, Spencer AJ, Davies MJ, Stewart JF. Influence of exposure to fluoridated water on socio economic inequalities in children's caries experience. Community Dent Oral Epidemiol 1996; 24: 89-100.

6. Jones CM, Worthington $\mathrm{H}$. Water fluoridation, poverty and tooth decay in 12-year-old children. J Dent 2000; 28 : 389-93.

7. Burt BA. Fluoridation and social equity. J Public Health Dent 2002; 62: 195-200.

8. Cangussu MCT, Narvai PC, Fernandes RC, Djehizian V. A fluorose dentária no Brasil: uma revisão crítica. Cad Saúde Pública 2002; 18: 7-15.

9. Riordan PJ. Perceptions of dental fluorosis. J Dent Res 1993; 72: 1268-74.

10. Lalumandier JA, Rozier RG. Parents' satisfaction with children's tooth color: fluorosis as a contributing factor. $J$ Am Dent Assoc 1998; 129: 1000-6.

11. Brasil. Ministério da Saúde. Secretaria de Atenção à Saúde. Departamento de Atenção Básica. Condições de saúde bucal da população brasileira 2002-2003: resultados principais. Brasília; 2004.

12. Moyses SJ, Moyses ST, Allegretti ACV, Argenta M, Werneck R. Fluorose dental: ficção epidemiológica? Rev Panam Salud Publica 2002; 12: 339-46.

13. Goldstein RE. A estética em Odontologia. $2^{\text {a }}$ ed. São Paulo: Santos; 2000.

14. Peres KG, Latorre MRDO, Peres MA, Traebert J, Panizzi M. Impacto da cárie e da fluorose dentária na satisfação com a aparência e com a mastigação de crianças de 12 anos de idade. Cad Saúde Pública 2003; 19: 323-30.

15. McKnight CB, Levy SM, Cooper SE, Jakobsen JR, Warren JJ. A pilot study of dental students' perceptions of computer-generated mild dental fluorosis compared to other conditions. J Public Health Dent 1999; 59: 18-23.
16. Hawley GM, Ellwood RP, Davies RM. Dental caries, fluorosis and the cosmetic implications of different TF scores in 14-year-old-adolescentes. Community Dental Health 1996; 13: 189-92.

17. Edwards M, McPherson SMD, Simmons ER, Gilmour WH, Stephen KW. An assessment of teenagers' perceptions of dental fluorosis using digital simulation and web-based testing. Community Dent Oral Epidemiol 2005; 33: 298-306.

18. Martins CC, Pinheiro NR, Paiva SM. Percepção da fluorose dentária sob a ótica dos pais de crianças portadoras: até que ponto o comprometimento estético dos dentes é aceitável? J Bras Odontopeditr Odontol Bebê 2003; 6: 413-8.

19. Riordan PJ. Specialist clinician's perceptions of dental fluorosis. ASDC J Dent Child 1993: 60: 315-20.

20. Shulman JD, Maupomé G, Clark C, Levy SM. Perceptions of desirable tooth color among parents, dentists and children. J Am Dent Assoc 2004; 135: 595-604.

21. Levy SM, Warren JJ, Jakobsen JR. Follow-up study of dental students' esthetic perceptions of mild dental fluorosis. Community Dent Oral Epidemiol 2002; 30: 24-8.

22. Clark DC. Evaluation of aesthetics for the different classifications of the Tooth Surface Index of Fluorosis. Community Dent Oral Epidemiol 1995; 23: 80.

23. Clark DC, Berkowitz J. The influence of various fluoride exposures on the prevalence of esthetic problems resulting from dental fluorosis. J Public Health Dentistry 1997; 57: 144.

24. Organização Mundial da Saúde. Levantamentos básicos em saúde bucal. 4a ed. São Paulo: Santos; 1999

25. McKnight CB, Levy SM, Cooper SE, Jakobsen JR. A pilot study of esthetic perceptions of dental fluorosis vs. selected other dental conditions. ASCD J Dent Child 1998; 65: 233-8.

26. Pietilä T, Pietilä I. Parents' views on their own child's dentition compared with an orthodontist's assessment Eur J Orthod 1994; 16(4): 309-16.

27. Lynch CD, McConnel RJ. The use of microabrasion to remove discolored enamel: a clinical report. J Prosthet Dent 2003; 90: 417-19.

Recebido em: 29/11/07 Versão final reapresentada em: 05/06/08 Aprovado em: 16/07/08 